\title{
87. The Comparison of Chromosomes among Different Species in Triticum.
}

\author{
By Fuyuwo Kagawa.
}

Utsunomiya Agricultural College.

(Rec. April 15, 1927. Comm. by S. KIKKAWA, M.I.A., May 12, 1927.)

The somatic chromosomes of different species in Triticum have been compared according to their length as well as to the number and position of constrictions.

The root tips have been treated in the dilute aqueous solutions of chloralhydrate and of ether, and this has been followed by washing in running water. These root tips have been kept in moist space for a certain period of time, and then they have been fixed. In the material fixed without chloralization and etherization the chromosomes of Triticum are observed as long, irregularly curved bands. But in the treated root tips the chromosomes appear much stouter and thicker, many of them lying almost perpendicular to the microscopic axis. The curvature of chromosomes is much simpler, and they often appear as almost straight rods. Consequently the estimation of chromosome length is more easily done than in ordinarily fixed material. In the chloralized and etherized root tips the constriction of chromosomes appear much clearer than those of ordinarily fixed ones. Many of these constrictions can be observed only in the treated root tips, but not in the normal roots. The precise measuring of the length of chromosomes and of chromosome parts divided by the constrictions, which is important for the exact discrimination of chromosomes, has not yet been made. But if a large difference exists in length in these respects, they are easily recognized in favourable figures in such experimental material as that stated above. So the classification and identification of chromosomes thus made without precise measuring may afford us the approximately exact results.

In this way the 14 somatic chromosomes of Triticum monocoscum, a diploid species, have been classifield into 5 groups $\mathrm{I}-\mathrm{V}$, and they may be presented by the formula, $2 \mathrm{I}+2 \mathrm{II}+2 \mathrm{III}+2 \mathrm{IIII}+6 \mathrm{~V}$. In the formula the number in each group indicates the number of chromosomes con- 
tained therein. The chromosomes belonging to groups I, II, III and IIII have 2 constrictions at the definite positions characteristic to each group. The chromosomes of group $\mathrm{V}$ have one constriction, and the relative position of this and the length of chromosomes seem to be not the same in all chromosomes of the group. Precise measuring in these respects may possibly make further classification in this group.

In T. polonicum, a tetraploid species, the somatic chromosome number being 28 , the chromosomes may be classified into at least 8 groups. In each of 6 groups out of 8 the chromosomes present 2 constrictions at the relative positions definite to the group. In the other 2 groups the chromosomes show one constriction, its relative position being different in different groups. Among 7 groups out of 8 there are at least 5 groups of chromosomes which, judging by their state of constrictions, could not be observed in T. monococcum. In any of the 7 chromosome groups there has been observed no case in which more than 3 homologous chromosomes are ascertained to appear in a nucleus. 2 is the maximum number of the homologous chromosomes in 5 groups out of 7 , and in the other 2 groups 3 was found as such in a few nuclei, though with some doubt. It may be assumed that in these 5 groups chromosomes exists in one pair each, and in the other 2 groups the question remains whether the number of pairs is 1 or 2 . At any rate these 28 chromosomes do not present the 4 -fold multiplication of any ground set of chromosomes so far as the conditions of constrictions are concerned.

In $T$. dicoccum, also a tetraploid species, 28 chromosomes may be classified into at least 10 groups. The chromosomes in each of 7 groups out of 10 have 2 constrictions at the positions definite to the group. In the other 3 groups one constriction is observed in each chromosome at the point characteristic to the group. Among 9 groups out of 10 there have been observed at least 7 groups of chromosomes which, according to the state of constrictions, could not be found in T. morococcum. The number of homologous chromosomes in a nucleus is always less than 4 , the number corresponding to tetraploidy. The maximum number of homologous chromosomes has been observed as 2 except in one group in which 3 was found as such in a few cases, though with some uncertainty.

Some chromosomes in $T$. polonicum and $T$. dicoccum present almost the same feature as to the constriction as were observed in T. monococcum, but the number of these chromosomes in a nucleus in these tetraploid species is always within the scope of 2 , not the multiplication of the number of corresnonding chromosomes in $T$. monococcum. 
In T. vulgare, a hexaploid species, the somatic chromosome number being 42 , the chromosomes may be classified into at least 9 groups. In 4 groups out of 9 , chromosomes have 2 constrictions at the relative positions definite to the group. In the other 5 groups the chromosomes show one constriction each, the relative position of which being different by groups. Among these 8 groups out of 9 there have been observed at least 7 groups of chromosomes which, according to the conditions of constrictions, could not be found in T. monococcum. The 6 -fold multiplication of a ground set of chromosomes is not present in $T$. vulgare. The number of homologous chromosomes in a nucleus is always less than 6 , the number corresponding to hexaploidy. 2 is the maximum number observed as such.

In all of the above stated tetraploid and hexaploid species the chromosomes in one of the groups in which one constriction is located in a chromosome, can be further classified by the measurement of the relative position of constrictions and also of the length of chromosomes.

In $T$. polonicum, $T$. dicoccum and in $T$. vulgare it was observed that between certain chromosomes in a uncleus the difference of chromosome length is much larger than that found in T. monococcum.

In $T$. monococcum, $T$. polonicum, $T$. dicoccum and $T$. vulgare the chromosome numbers show the multiple series, the basis of which being 7, which is the haploid chromosome number in T. monococcum. But, as is clear in the above article, the chromosome contents of these tetraploid-and hexaploid species are not the multiplication of that in $T$. monococcum so far as the state of constriction and the length of chromosomes are concerned. So that these 4 Triticum species do not present the polyploidy in the sense that the polyploidy is a phenomenon in which the multiplication of one ground set of chromosomes is concerned. These polyploid species may not have been phylogenetically produced by the multiplication of any ground set of chromosomes, including that of $T$. monococcum, though the possibility and the modes of the multiplication have been discussed by investigators in a number of ways. It seems probable that there are contained in the formative causes of polyploid species in Triticum the crosses between certain ancestral forms having different chromosome contents with each other and the special chromosome behavior which followed these crosses.

The expense of the present study has partly been defrayed by a grant given by The Imperial Academy. 